\title{
Materialization of Fragmented Views in Multidimensional Databases *
}

\author{
Matteo Golfarelli ${ }^{\text {a,*}}{ }^{\text {, Vittorio Maniezzo }}{ }^{\text {b }}$, Stefano Rizzi ${ }^{\text {a }}$ \\ a DEIS, University of Bologna, Viale Risorgimento 2, 40136 Bologna - Italy \\ ${ }^{\mathrm{b}}$ Computer Science Dept., University of Bologna, Mura Anteo Zamboni, 7, 40127 \\ Bologna - Italy
}

\begin{abstract}
A multidimensional database can be seen as a collection of multidimensional cubes centered on facts of interest. In the context of multidimensional databases implemented on relational DBMSs, the most effective technique to enhance performances consists in materializing redundant aggregates called views. In the classical approach to materialization, each view includes all and only the measures of the cube it aggregates. In this paper we investigate the benefits of materializing views in vertical fragments, each possibly including measures from different cubes, aimed at minimizing the workload response time. In fact, the measures of a single cube are not necessarily always requested together in queries; on the other hand, drillacross queries involve measures taken from two or more cubes. We formalize the fragmentation problem as a 0-1 integer linear programming problem, which is then solved by means of a standard integer programming solver to determine the optimal fragmentation for a given workload. Finally, we demonstrate the usefulness of fragmentation by presenting a large set of experimental results based on the TPC-H benchmark.
\end{abstract}

Key words: Data warehousing, Logical design, View materialization

\footnotetext{
‡ This research was partially supported by MURST - Interdata Project.

* Corresponding author. Tel.: +39-0547-642862; fax: +39-0547-610054

Email addresses: mgolfarelli@deis unibo.it (Matteo Golfarelli), maniezzo@csr.unibo.it (Vittorio Maniezzo), srizzi@deis.unibo.it (Stefano Rizzi).
} 


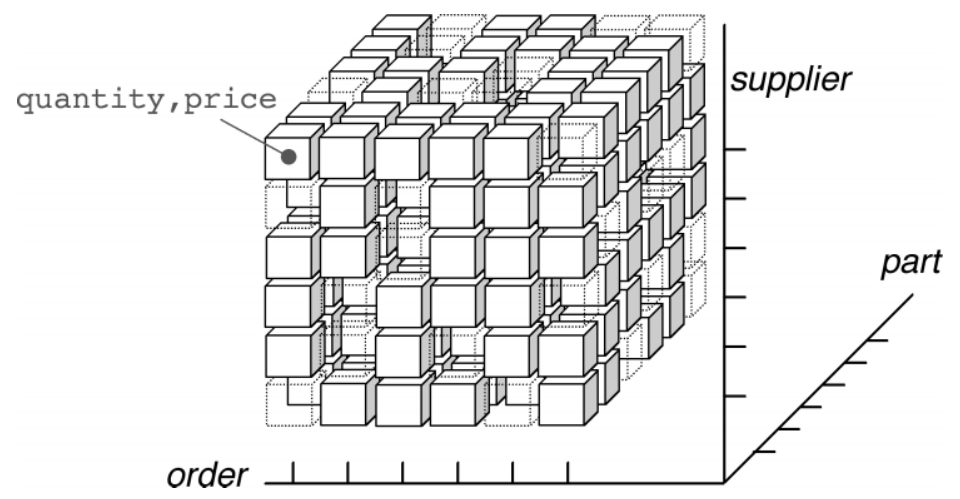

Fig. 1. A sample cube for the line items in an order.

\section{Introduction}

Recently, multidimensional databases have gathered wide research and market interest as the core of decision support applications such as data warehouses. A multidimensional database (MD) can be seen as a collection of multidimensional cubes centered on facts of interest (for instance, the line items in the orders issued by a company). A cube is composed by cells which contain a set of numerical measures conveying useful information for the decision process (for instance, the price and the quantity of a line item). The cube $d i$ mensions are attributes which represents possible dimensions for analysis (for instance, products, suppliers and orders) [12]. From the logical point of view, the set of dimensions functionally determines every measure (see Figure 1). Furthermore, each dimension may be associated to a hierarchy of attributes which describe it (for instance, the city and the nation of a supplier).

An MD implemented on a relational DBMS is usually organized according to the so-called star scheme [14], in which each cube is represented by one fact table storing the measures and one denormalized dimension table for each dimension of analysis. The primary key of the fact table is a set of foreign keys, each referencing the (usually surrogate) key of a dimension table. Besides the key, each dimension table contains all the attributes which describe the corresponding dimension.

In MDs, the response to the workload is improved by materializing a set of views which store the pre-computed results to frequent/critical queries, thus introducing some degree of data redundancy. Since the basic mechanism to extract useful information from elemental data in MDs is aggregation, a view on a cube is typically defined by a SQL query which groups data by a set of attributes taken from dimension tables (grouping set, for instance including order date and part type) and computes summarized values for measures by means of some aggregation operators (see Figure 2). Even views are organized into star schemes. In the following, with a slight abuse in terminology, we 


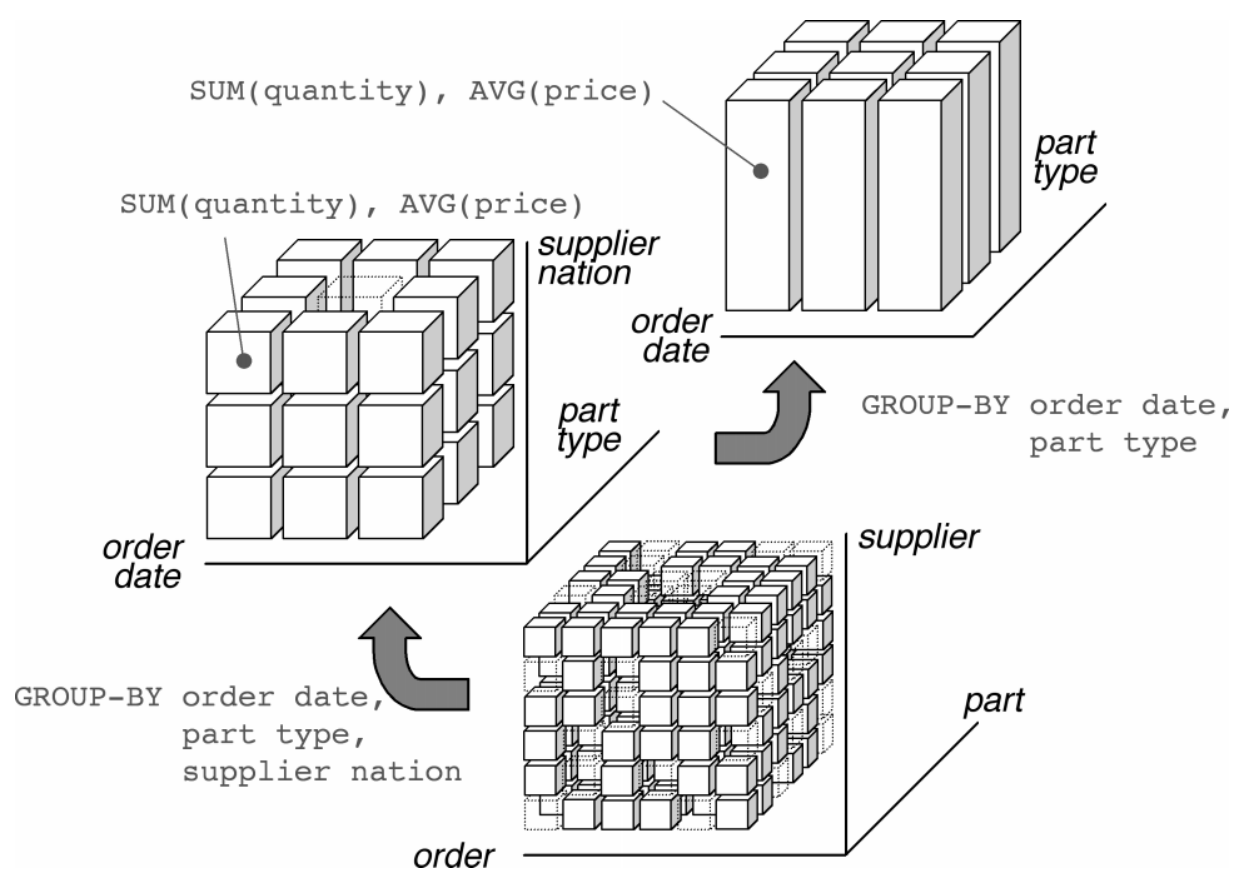

Fig. 2. Two views on the line item cube.

will call views all the star schemes for a cube: the one storing elemental data (primary view) and those containing aggregated values (secondary views).

Since pre-computing and storing all the possible secondary views is infeasible, several techniques have been proposed to select the subset to materialize in order to optimize the response to the workload (e.g, [1,13,27,24]). In these approaches, the views to be materialized on a cube include all its measures, without considering which measures are required together by the queries in the workload; hence, we will globally refer to them as all-or-nothing approaches.

In this paper we investigate how the response to the workload can be enhanced by materializing views in vertical fragments, each including only a subset of measures. The most evident motivations for applying vertical fragmentation techniques to views in MDs can be stated as follows:

- As compared to operational databases, in MDs the benefits of fragmentation are further enhanced by the multiple query execution plans due to the presence of redundant secondary views. These benefits are particularly relevant if the MD is implemented on a parallel architecture; if disk arrays are adopted and fragmentation is coupled with an allocation algorithm, the queries requiring multiple fragments allocated on different disks can be effectively parallelized $[17,19]$.

- While every measure of each cube must be stored in its elemental form to avoid loss of information, the same is not true for aggregated values. Thus, depending on the workload, some measures may be not materialized at all in aggregated fragments, leading to space saving as compared to all- 
or-nothing materialization. When a space constraint is posed, this saving can be profitably employed to store other useful fragments.

- Partitioning a view in two or more fragments entails no significant storage overhead: in fact, on the one hand, surrogate keys require few bytes to be stored; on the other, though on primary views the number of dimensions may exceed the number of measures, this is less likely on secondary views for two reasons: firstly, they may also include derived measures and support measures for non-distributive aggregation operators [6,9]; secondly, within a coarse grouping set, one or more dimensions may be completely grouped (in this case, the corresponding foreign key is dropped from the key of the view).

Vertical fragmentation for MDs has already been proposed in the literature $[4,5,17]$; in this paper we give the following new contributions:

- Optimality. In the other approaches, no suggestions on how to determine a good fragmentation were given. In this paper, the fragmentation problem is formalized as a 0-1 integer programming (IP) problem, which is then solved by means of a standard IP solver. Given a core workload, a constraint on the global disk space for materialization and a function to estimate the cost for executing each query on each fragment, an optimal fragmentation is thus produced.

- Generality. Instead of first determining the optimal set of views and then fragmenting them, we propose to directly determine the optimal fragments to be materialized from the workload. This one-step approach avoids losing in overall optimality by allocating useless fragments. Besides, our approach completely subsumes the all-or-nothing approach: in fact, if all the queries in the workload require all and only the measures of a single cube, only unfragmented views are determined.

- Drill-across. In our approach, each fragment includes measures taken from one or more cubes, aggregated on the same grouping set. Thus, as compared to all-or-nothing materialization, fragmentation may achieve the goal of unifying two or more cubes into a single fragment besides that of partitioning a cube into two or more fragments. While partitioning may be useful whenever only a subset of the attributes is typically required by each query, unification may be convenient when the workload is significantly affected by drill-across queries, i.e. queries formulated on measures belonging to different cubes.

- Testing. The results of a large set of experimental results is reported in the paper in order to quantify and discuss the benefits of fragmentation from different points of view.

The paper is structured as follows. In Section 2 we introduce the basic definitions and notations for the paper. In Section 3 the fragmentation problem is motivated and formalized as a 0-1 IP problem. Section 4 presents a large 
set of experimental results based on the TPC-H benchmark. In Section 5 the related work is surveyed, while Section 6 draws the conclusions. Appendix A describes the cost function used for optimization.

\section{Background}

This section introduces some necessary background knowledge for the paper. In particular, Section 2.1 introduces the basics on cube schemes, Section 2.2 discusses the assumptions made on the workload for the MD, and Section 2.3 identifies the candidate views for materialization.

\subsection{Cubes}

Definition 1 (Cube Scheme) $A$ cube scheme $\mathcal{C}$ is defined by a set of dimensions, $G b y(\mathcal{C})$, and a set of measures, Meas $(\mathcal{C})$. If $n$ is the number of dimensions in $\mathcal{C}$, we will say $\mathcal{C}$ is n-dimensional.

Typically, each dimension is associated with a set of attributes which describe it; these attributes are organized into a directed tree, called hierarchy, whose root is the dimension and whose arcs represent functional dependencies. A cube instance of a cube scheme is a partial function which maps from the Cartesian product of the domains of dimensions to the Cartesian product of the domains of measures.

Definition 2 (Multidimensional Scheme) $A$ multidimensional scheme $\mathcal{D}$ is a set of cube schemes.

Example 1 The multidimensional scheme we will use as a working example derives from the TPC-H [20]; it includes two cube schemes, Lineltem $(L I)$ and PartSupplier $(P S)$, which respectively describe the composition of the orders issued from a company and the company supplies:

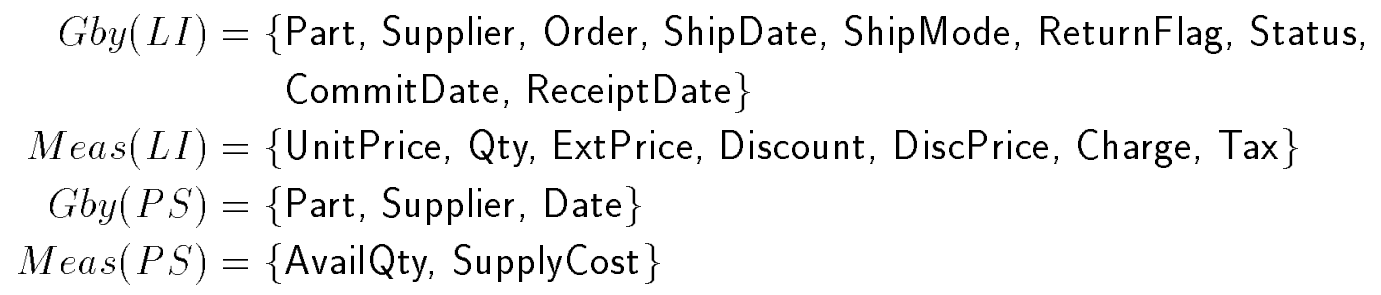

$L I$ and $P S$ are characterized by the hierarchies represented graphically in Figure 3 where, for instance, Supplier $\rightarrow$ SNation and SNation $\rightarrow$ SRegion. On re- 

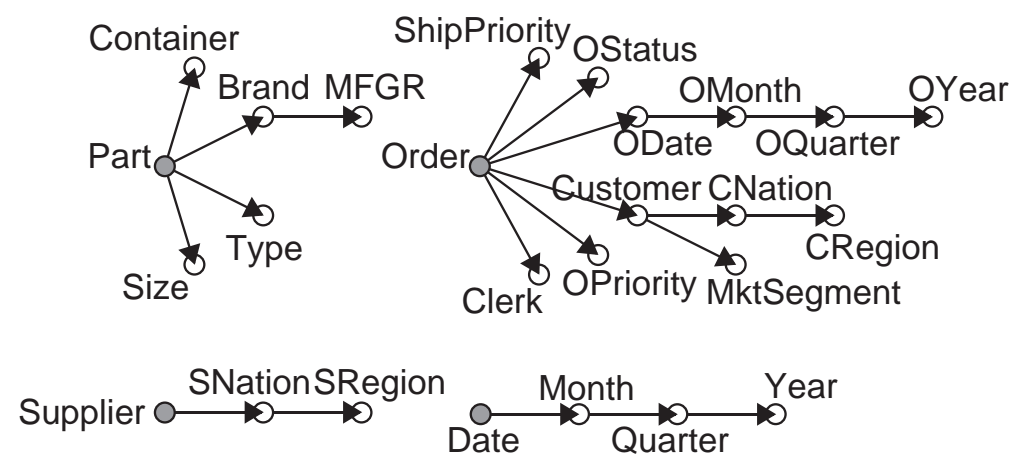

Fig. 3. Hierarchies in the $L I$ and $P S$ schemes (in gray the dimensions).

lational DBMSs, cube schemes are usually implemented through a star scheme [14]. The star scheme for $L I$ is:

PART(Part, Brand, MFGR, Type, Container, Size)

SUPPLIER(Supplier, SNation, SRegion)

ORDER(Order, ODate, OMonth, OQuarter, OYear, OWeek, Customer, CNation, CRegion, MktSegment, OPriority, OStatus, ShipPriority, Clerk)

LINEITEM(Part, Supplier, Order, ShipDate, ShipMode,

ReturnFlag,ReceiptDate, CommitDate, Status,

UnitPrice, Qty, ExtPrice, Discount, DiscPrice, Charge, Tax)

where LINEITEM is the fact table and the others are dimension tables. ${ }^{1}$

\subsection{The workload}

In principle, the workload for an MD is dynamic and unpredictable. Nevertheless, in agreement with several research papers (for instance [9,27]), we claim that a core workload can be determined a priori. In fact, on the one hand, the user typically knows in advance which kind of data analysis (s)he will carry out more often for decisional or statistical purposes; on the other, a substantial amount of queries are aimed at extracting summary data to fill standard reports. Furthermore, some commercial tools enable the workload to be monitored while the MD is operating: in this case, fragmentation could be carried out periodically considering the current workload in order to tune performances at best.

In the following we will consider the GPSJ class of queries, very common in OLAP applications. A GPSJ (Generalized Projection / Selection / Join) query is a selection over a generalized projection over a selection over a join,

\footnotetext{
$\overline{1}$ In the working example we will assume for simplicity that the primary key of each dimension table is a dimension. In practice, surrogate keys are typically introduced instead.
} 
where the generalized projection operator is an extension of duplicate eliminating projection which captures grouping and aggregation [11]. In a GPSJ query within the OLAP context, the join is the star join that relates the fact table and the dimension tables; the selection may be applied to both measures (non-key attributes of the fact table) and attributes (non-key attributes of dimension tables); the generalized projection groups the tuples on a set of attributes (grouping set) and determines which aggregated measures are returned. Given query $q$, we will denote with $G b y(q), \operatorname{Meas}(q), \operatorname{Sel}(q) \in[0 . .1]$, and Freq( $q)$, respectively, the grouping set of $q$, the measures it returns, its selectivity, and its frequency within the workload.

Example 2 On $L I$, the query asking for the total quantity of each medium polished part ordered from American suppliers is characterized by $G b y(q)=$ $\{$ SRegion, Part $\}$, Meas $(q)=\{$ Qty $\}$ and $\operatorname{Sel}(q)=0.01$ (assuming that 5 supplier regions and 20 part types are present, that attribute values are uniformly distributed and that selection predicates are independent, it is $\left.\operatorname{Sel}(q)=\frac{1}{5} \cdot \frac{1}{20}\right)$. Its SQL formulation on the star scheme defined in Example 1 is:

$\begin{array}{ll}\text { SELECT } & \text { S.SRegion, P.Part, SUM(LI.Qty) } \\ \text { FROM } & \text { LINEITEM AS LI, PART AS P, SUPPLIER AS S } \\ \text { WHERE } & \text { LI.Part = P.Part } \\ \text { AND } & \text { LI.Supplier = S.Supplier } \\ \text { AND } & \text { P.Type = 'Medium Polished' } \\ \text { AND } & \text { S.SRegion = 'USA' } \\ \text { GROUP BY S.SRegion, P.Part }\end{array}$

Given a cube scheme $\mathcal{C}$, let $\mathcal{G}_{\mathcal{C}}$ denote the set of the grouping sets of all the possible queries on $\mathcal{C}$. As observed in [1], the functional dependencies which relate the attributes of hierarchies in $\mathcal{C}$ induce a partial ordering, called roll$u p(\preceq)$, on $\mathcal{G}_{\mathcal{C}}$ : given two grouping sets $g_{i}$ and $g_{j}$, it is $g_{i} \preceq g_{j}$ iff $g_{j} \rightarrow g_{i}\left(g_{j}\right.$ functionally determines $g_{i}$ ). The roll-up ordering identifies a lattice in which the top element is $G b y(\mathcal{C})$ (the finest grouping set) and the bottom element is the empty grouping set, $\emptyset$ (the coarsest possible one). While $G b y(\mathcal{C})$ will be called the primary grouping set of $\mathcal{C}$, all the other grouping sets in $\mathcal{G}_{\mathcal{C}}$ will be called the secondary grouping sets of $\mathcal{C}$. 


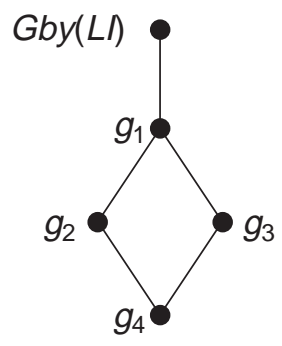

Fig. 4. Roll-up relationships between grouping sets on the $L I$ scheme.

Example 3 Some examples of grouping sets on $L I$ are

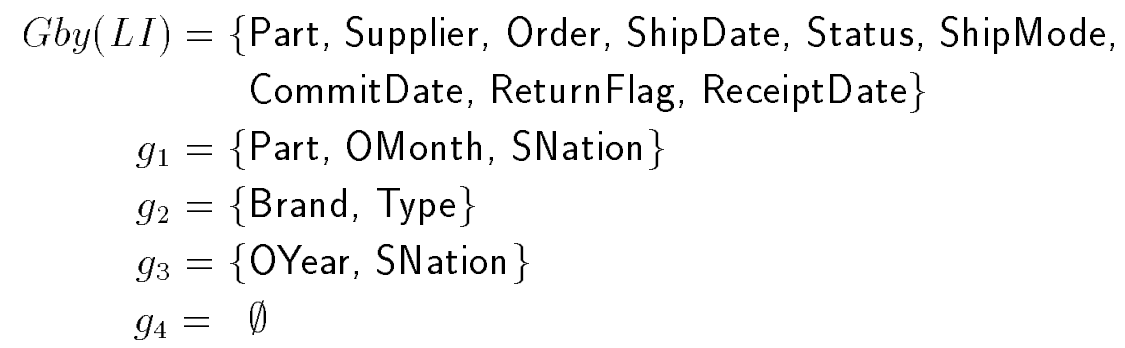

The roll-up relationships between them are summarized in Figure 4.

Though a large part of the queries the user formulates involve a subset of measures of a single cube scheme $(\operatorname{Meas}(q) \subseteq$ Meas $(\mathcal{C}))$, part of the queries may require comparing measures aggregated on the same grouping set but taken from distinct cube schemes; in the OLAP terminology, these are called drill-across queries.

Example 4 The query which compares the total available quantity and the total quantity sold for each part, characterized by $G b y(q)=\{\operatorname{Part}\}$, Meas $(q)=$ $\{$ AvailQty, Qty , and $\operatorname{Sel}(q)=1$, is a drill-across query on $L I$ and PS. Its SQL formulation is the following:

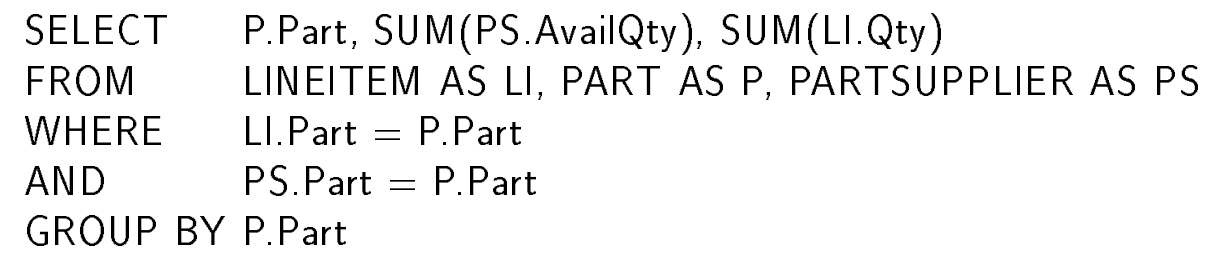

Given a query $q$ and a set of measures $M^{\prime} \subset \operatorname{Meas}(q)$, we will call the projection of $q$ on $M^{\prime}$ the query $q^{\prime}$ characterized by $G b y\left(q^{\prime}\right)=G b y(q), \operatorname{Sel}\left(q^{\prime}\right)=$ $\operatorname{Sel}(q)$, and Meas $\left(q^{\prime}\right)=M^{\prime}$. 


\subsection{Candidate views}

In MDs, the response to the workload is typically improved by materializing, besides the primary view storing elemental data, a set of secondary views which store the pre-computed results to frequent/critical queries. In the classical approaches to materialization, each view includes all and only the measures of one cube scheme; thus, in principle, each grouping set on a given cube scheme determines exactly one possible view to be materialized.

Several algorithms have been proposed to determine the set of materialized views which minimizes the workload cost by respecting a constraint posed on the total storing space. Most approaches $[1,5,13]$ start by determining the set of views which may potentially reduce the cost for executing the workload (candidate views) ${ }^{2}$; then, the views to be materialized are chosen by applying some (exact or heuristic) optimization technique to the set of candidate views. In particular, in [1] the authors supply a criterion for defining candidate views and prove that materializing a non-candidate view can never decrease the workload cost.

In the remainder we will assume that a set of candidate views has been determined for each cube scheme involved in the workload. To the best of our knowledge, no approach to materialization in the literature takes drill-across queries into account; on the other hand, since these queries often play a relevant role within the workload, it is necessary to involve them in the optimization process. Thus, in determining the candidate views, every drill-across query is substituted in the workload by its projections on the measures of the cube schemes involved.

Let $C$ and $(\mathcal{C})$ denote the set of grouping sets of the candidate views (candidate grouping sets) determined for cube scheme $\mathcal{C}$. For each cube scheme, every measure must be materialized at the primary grouping set (i.e. in its nonaggregated form); thus, $G b y(\mathcal{C}) \in \operatorname{Cand}(\mathcal{C})$ for every $\mathcal{C}$.

\section{Materialization of fragmented views}

While in the classical approach to materialization each view is univocally characterized by its grouping set and by the cube scheme it is computed from, in our approach a view (which will be more properly called fragment) is characterized by its grouping set and by a set of measures belonging to the

$\overline{2}$ For instance, a view whose grouping set is coarser than the grouping sets of all the queries in the workload is never useful, thus it is not candidate. 
cube schemes in the multidimensional scheme.

Definition 3 (Fragment) Given multidimensional scheme $\mathcal{D}$, a fragment $\mathcal{F}$ on $\mathcal{D}$ is defined by a grouping set $G$ by $(\mathcal{F})$ and by a set of measures Meas $(\mathcal{F})$, where $G$ by $(\mathcal{F}) \in \mathcal{G}_{\mathcal{C}_{k}}$ for each $\mathcal{C}_{k} \in \mathcal{D}$ such that Meas $(\mathcal{F}) \cap \operatorname{Meas}\left(\mathcal{C}_{k}\right) \neq \emptyset$.

In terms of relational implementation, fragment $\mathcal{F}$ corresponds to a fact table having the attributes in $G b y(\mathcal{F})$ as the key and the measures in Meas $(\mathcal{F})$ as non-key attributes.

Example 5 The fact table for fragment $\mathcal{F}$ with $G b y(\mathcal{F})=\{\operatorname{Part}\}$ and $\operatorname{Meas}(\mathcal{F})=$ $\{$ AvailQty, Qty\} has scheme

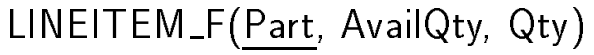

and is defined by the query in Example 4.

Definition 4 (Fragmentation) Let Cand $\left(\mathcal{C}_{k}\right)$ be the candidate grouping sets for $\mathcal{C}_{k} \in \mathcal{D}$. A fragmentation on $\mathcal{D}$ is a set $\mathcal{H}$ of fragments with the following properties:

(1) Consistent. A fragment always corresponds to a candidate grouping set:

$$
\begin{aligned}
\forall \mathcal{F}_{i} \in \mathcal{H}, \forall m_{s} \in & \operatorname{Meas}\left(\mathcal{F}_{i}\right) \\
& \exists \mathcal{C}_{k} \in \mathcal{D}: \operatorname{Gby}\left(\mathcal{F}_{i}\right) \in \operatorname{Cand}\left(\mathcal{C}_{k}\right) \wedge m_{s} \in \operatorname{Meas}\left(\mathcal{C}_{k}\right)
\end{aligned}
$$

(2) Lossless. Each measure of every cube scheme is materialized at least at its primary grouping set:

$$
\begin{aligned}
\forall \mathcal{C}_{k} \in \mathcal{D}, \forall m_{s} \in & \operatorname{Meas}\left(\mathcal{C}_{k}\right) \\
& \exists \mathcal{F}_{i} \in \mathcal{H}: G b y\left(\mathcal{F}_{i}\right)=G b y\left(\mathcal{C}_{k}\right) \wedge m_{s} \in \operatorname{Meas}\left(\mathcal{F}_{i}\right)
\end{aligned}
$$

(3) Non-redundant. No overlap between fragments is allowed:

$$
\forall \mathcal{F}_{i}, \mathcal{F}_{j} \in \mathcal{H}\left(\operatorname{Gby}\left(\mathcal{F}_{i}\right)=\operatorname{Gby}\left(\mathcal{F}_{j}\right)\right) \Rightarrow\left(\operatorname{Meas}\left(\mathcal{F}_{i}\right) \cap \operatorname{Meas}\left(\mathcal{F}_{j}\right)=\emptyset\right)
$$

It should be noted that several fragments, even defined on different grouping sets, may be necessary to solve a given query. Given a subset of fragments $\mathcal{H}^{\prime} \subset \mathcal{H}$, query $q$ can be answered on $\mathcal{H}^{\prime}$ iff

$$
\forall m_{s} \in \operatorname{Meas}(q) \exists \mathcal{F}_{i} \in \mathcal{H}^{\prime}: m_{s} \in \operatorname{Meas}\left(\mathcal{F}_{i}\right) \wedge G b y(q) \preceq G b y\left(\mathcal{F}_{i}\right)
$$

Due to constraint (2), every possible query on $\mathcal{D}$ can be answered on $\mathcal{H}$. If $q$ necessarily requires two or more fragments (since no fragment includes all the measures in Meas $(q)$ ), it must be solved by first solving all its projections 
on the measures of the single fragments, then performing a join between the results (the join attributes are those in $G b y(q)$ ).

In the remainder of this section we face the problem of determining the fragmentation which minimizes the workload cost satisfying a given constraint on the global space for materialization. After emphasizing the motivations for fragmentation by proposing a quantitative example on the TPC-H benchmark, we will introduce the problem formulation and propose a mathematical programming approach to determine the optimal fragmentation.

\subsection{Fragmentation vs. classical materialization: an example}

In the all-or-nothing approach to materialization, selecting a candidate grouping set $g \in \operatorname{Cand}(\mathcal{C})$ during the optimization process means materializing a view with grouping set $g$ and measures $\operatorname{Meas}(\mathcal{C})$. As a result, each view includes measures which describe the same cube scheme but, within the workload, may be often requested separately. We argue that the system overall response to the workload may be increased by materializing fragments which include only the measures which typically appear together within the queries.

Moreover, drill-across queries are typically solved by joining views defined on different cube schemes. In our approach, the access costs for these queries may be decreased by materializing fragments which include measures taken from different cube schemes, aggregated on the same grouping set.

Let the workload on $L I$ and PS include three queries defined as follows:

$$
\begin{aligned}
\operatorname{Meas}\left(q_{1}\right) & =\{\text { DiscPrice, Charge, Tax }\} \\
\text { Gby }\left(q_{1}\right) & =\{\text { Brand, Supplier }\} \\
\text { Meas }\left(q_{2}\right) & =\{\text { UnitPrice, Qty, ExtPrice, Discount }\} \\
\text { Gby }\left(q_{2}\right) & =\{\text { Part, Supplier, ShipDate }\} \\
\text { Meas }\left(q_{3}\right) & =\{\text { Qty, AvailQty }\} \\
\text { Gby }\left(q_{3}\right) & =\{\text { Part, Supplier }\}
\end{aligned}
$$

While $q_{1}$ and $q_{2}$ are formulated on $L I, q_{3}$ is a drill-across query on $L I$ and $P S$; all selectivities are assumed equal to 1 . The candidate grouping sets for this workload are

$$
\begin{aligned}
C a n d(L I) & =\left\{G b y(L I), G b y\left(q_{1}\right), G b y\left(q_{2}\right), G b y\left(q_{3}\right)\right\}, \\
C \text { and }(P S) & =\left\{G b y(P S), G b y\left(q_{3}\right)\right\}
\end{aligned}
$$

Using a classical approach, the possible secondary views to materialize are $\mathcal{V}_{1}$, 
Table 1

Execution costs; a dash denotes that a query cannot be executed on a view/fragment, the costs in parentheses denote that not all the measures required by the query are found on the fragment.

\begin{tabular}{c|rrr}
\hline View/fragment & $q_{1}$ & $q_{2}$ & $q_{3}$ \\
\hline $\mathcal{V}_{1}$ & 2084 & - & - \\
$\mathcal{V}_{2}$ & 54932 & 54932 & $(54932)$ \\
$\mathcal{V}_{3}$ & 6934 & - & $(6934)$ \\
$\mathcal{V}_{4}$ & - & - & $(2051)$ \\
$\mathcal{F}_{1}$ & 1028 & - & - \\
$\mathcal{F}_{2}$ & - & 35157 & $(35157)$ \\
$\mathcal{F}_{3}$ & - & - & 2051 \\
$\mathcal{F}_{4}$ & - & 38086 & 38086 \\
$\mathcal{F}_{5}$ & - & - & $(1172)$ \\
\hline
\end{tabular}

$\mathcal{V}_{2}, \mathcal{V}_{3}$, and $\mathcal{V}_{4}$ characterized by

$$
\begin{aligned}
\operatorname{Meas}\left(\mathcal{V}_{1}\right) & =\operatorname{Meas}\left(\mathcal{V}_{2}\right)=\operatorname{Meas}\left(\mathcal{V}_{3}\right) \\
& =\{\text { UnitPrice, Qty, ExtPrice, Discount, DiscPrice, Charge, Tax }\}, \\
\operatorname{Meas}\left(\mathcal{V}_{4}\right) & =\{\text { AvailQty, SupplyCost }\} \\
G b y\left(\mathcal{V}_{1}\right) & =G b y\left(q_{1}\right), \\
G b y\left(\mathcal{V}_{2}\right) & =G b y\left(q_{2}\right), \\
G b y\left(\mathcal{V}_{3}\right) & =G b y\left(\mathcal{V}_{4}\right)=G b y\left(q_{3}\right)
\end{aligned}
$$

Consider now the five fragments $\mathcal{F}_{1}, \ldots, \mathcal{F}_{5}$ defined as follows:

$$
\begin{aligned}
\text { Meas }\left(\mathcal{F}_{1}\right) & =\{\text { DiscPrice, Charge, Tax }\}, \\
\operatorname{Gby}\left(\mathcal{F}_{1}\right) & =G b y\left(q_{1}\right) ; \\
\text { Meas }\left(\mathcal{F}_{2}\right) & =\{\text { UnitPrice, Qty, ExtPrice, Discount }\}, \\
\operatorname{Gby}\left(\mathcal{F}_{2}\right) & =\text { Gby }\left(q_{2}\right) ; \\
\text { Meas }\left(\mathcal{F}_{3}\right) & =\{\text { Qty, AvailQty }\}, \\
G b y\left(\mathcal{F}_{3}\right) & =G b y\left(q_{3}\right) ; \\
\operatorname{Meas}\left(\mathcal{F}_{4}\right) & =\{\text { UnitPrice, Qty, ExtPrice, Discount,AvailQty }\}, \\
\operatorname{Gby}\left(\mathcal{F}_{4}\right) & =\text { Gby }\left(q_{2}\right) ; \\
\operatorname{Meas}\left(\mathcal{F}_{5}\right) & =\{\text { AvailQty }\}, \\
\operatorname{Gby}\left(\mathcal{F}_{5}\right) & =\text { Gby }\left(q_{3}\right)
\end{aligned}
$$

The costs for executing each query on each view and fragment are summarized in Table 1. The function cost used expresses the number of disk pages in which the tuples required to answer the query are contained, and is detailed in Appendix A. In Table 2 some materialization solutions are compared in 
Table 2

Comparison of materialization solutions.

\begin{tabular}{l|rr}
\hline Mater. views $/$ fragments & Size (MB) & Workload cost (pages) \\
\hline $\mathcal{H}_{1}=\left\{\mathcal{V}_{2}, \mathcal{V}_{4}\right\}$ & 456 & 166847 \\
$\mathcal{H}_{2}=\left\{\mathcal{V}_{1}, \mathcal{V}_{2}, \mathcal{V}_{4}\right\}$ & 473 & 113999 \\
$\mathcal{H}_{3}=\left\{\mathcal{V}_{2}, \mathcal{V}_{3}, \mathcal{V}_{4}\right\}$ & 511 & 70851 \\
$\mathcal{H}_{4}=\left\{\mathcal{V}_{1}, \mathcal{V}_{2}, \mathcal{V}_{3}, \mathcal{V}_{4}\right\}$ & 528 & 66001 \\
$\mathcal{H}_{5}=\left\{\mathcal{F}_{1}, \mathcal{F}_{2}, \mathcal{F}_{3}\right\}$ & 306 & 38236 \\
$\mathcal{H}_{6}=\left\{\mathcal{F}_{1}, \mathcal{F}_{4}\right\}$ & 313 & 77200 \\
$\mathcal{H}_{7}=\left\{\mathcal{F}_{1}, \mathcal{F}_{2}, \mathcal{F}_{5}\right\}$ & 299 & 72514 \\
\hline
\end{tabular}

terms of total disk space occupied and workload cost; primary views are not considered, assuming that they will be materialized within all solutions. In particular, materializing three subsets of fragments is compared to materializing four subsets of views.

It clearly appears how fragmentation may reduce the overall workload cost since:

- While each view includes all the measures in its cube scheme, each fragment only includes those measures which, aggregated at the given grouping set, are useful for the workload.

- Materializing small fragments instead of large views leads to decreasing the query cost both directly, since smaller fact tables are to be read, and indirectly, since the storage space saved can be used to materialize extra fragments.

- Including measures from different cube schemes in the same fragment reduces the cost of drill-across queries since no join between fact tables is required.

- The extra space required by key duplication quickly decreases when the grouping set decreases; it is further reduced when junk dimensions ${ }^{3}$ are present.

- The effectiveness of fragmentation for MDs may be higher than for operational non-redundant databases; in fact, in MDs the presence of fragments storing the same measures at different levels of aggregation makes multiple solutions possible.

$\overline{3}$ In [14], the term junk dimensions is used to denote the case in which two or more hierarchies are represented jointly within the same dimension table. 


\subsection{Problem formulation}

In this section we propose the Vertical Fragmentation Problem (VFP) as a combinatorial optimization problem and discuss a possible IP formulation.

Recent IP results, both on exact [10] and heuristic [16,21] techniques, have in fact reached a point where even complex real-world problems can be solved in acceptable CPU time. It is thus worthwhile an investigation of efficient IP formulations, as these provide a key to directly access robust and effective solution techniques.

We begin by observing that, while in principle VFP should be solved for the whole set of cube schemes in the multidimensional scheme, in practice it may be convenient to mix measures belonging to two different cube schemes in the same fragment only if there is at least one candidate grouping set $g_{j}$ common to both cube schemes, and the workload includes at least one drillacross query on them which can be answered on $g_{j}$. In most cases, this allows to dramatically cut the complexity by adopting a simple divide-and-conquer approach and applying fragmentation separately on subsets of cube schemes based on partial workloads. In the following, $\mathcal{D}$ will stand for one of these subsets.

Given a workload, the measures of each cube scheme in $\mathcal{D}$ can be partitioned into subsets (minterms) such that all the measures in a minterm are requested together by at least one query and do not appear separately in any other query. We call terms the sets of measures, even of different cube schemes, recursively defined as follows:

(1) a minterm is a term;

(2) the union of two terms including measures required from the same query is a term.

For instance, on $L I$, given $Q=\left\{q_{1}, q_{2}, q_{3}\right\}$ where

$$
\begin{aligned}
& \text { Meas }\left(q_{1}\right)=\{\text { UnitPrice, Qty, ExtPrice, Discount }\}, \\
& \text { Meas }\left(q_{2}\right)=\{\text { UnitPrice, Qty, DiscPrice, Charge }\} \\
& \text { Meas }\left(q_{3}\right)=\{\text { DiscPrice, Charge, Tax }\}
\end{aligned}
$$

the minterms are:

$$
\begin{aligned}
& m t_{1}=\{\text { UnitPrice, Qty }\}, \\
& m t_{2}=\{\text { ExtPrice, Discount }\}, \\
& m t_{3}=\{\text { DiscPrice, Charge }\}, \\
& m t_{4}=\{\text { Tax }\}
\end{aligned}
$$




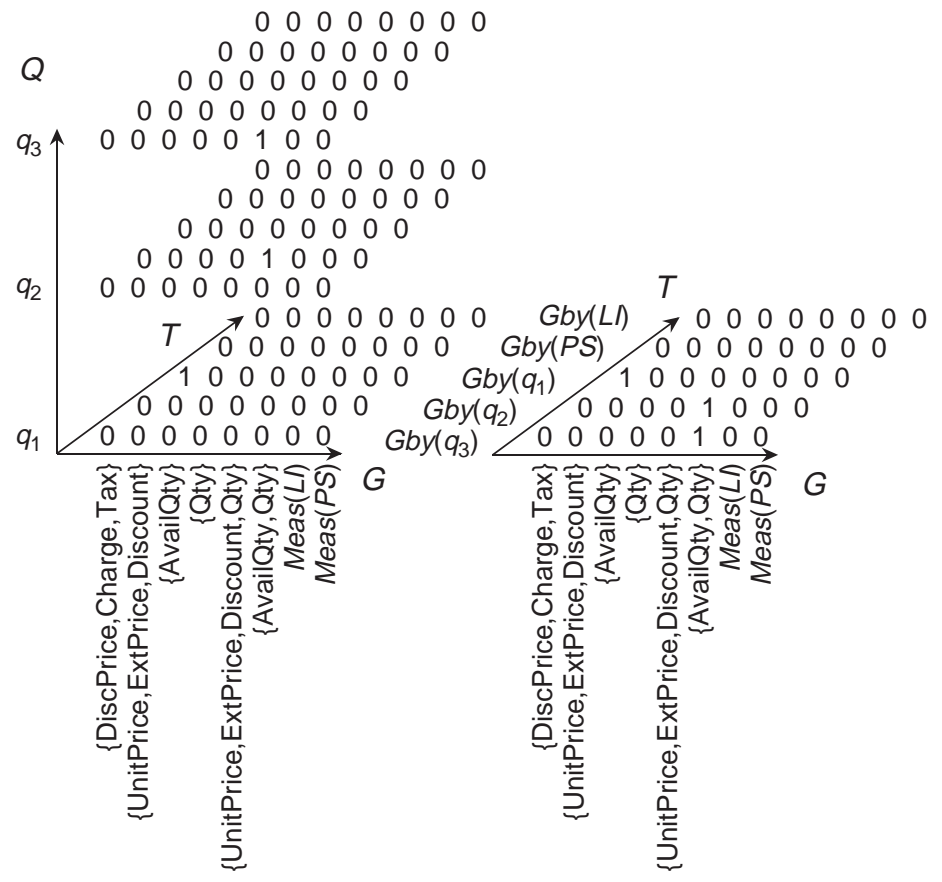

Fig. 5. Sets of the $x_{i j k}$ (left) and $y_{j k}$ (right) for fragmentation $\mathcal{H}_{5}$ in Section 3.1.

The corresponding set of terms is:

$$
\begin{gathered}
T=\left\{m t_{1}, m t_{2}, m t_{3}, m t_{4}, m t_{1} \cup m t_{2}, m t_{1} \cup m t_{3}, m t_{3} \cup m t_{4},\right. \\
\left.m t_{1} \cup m t_{2} \cup m t_{3}, m t_{1} \cup m t_{3} \cup m t_{4}, \operatorname{Meas}(L I)\right\}
\end{gathered}
$$

Given the multidimensional scheme $\mathcal{D}$ and workload $Q$, let $G=\bigcup_{\mathcal{C} \in \mathcal{D}} \operatorname{Cand}(\mathcal{C})$ be the set of all candidate grouping sets and $T$ the set of all terms. In the following we will show how VFP can be modeled by expressing linear IP constraints over two sets of $0-1$ binary variables, $\left\{x_{i j k}\right\}$ and $\left\{y_{j k}\right\}$, whose indexes correspond to the indexes of the queries $q_{i} \in Q$, of the grouping sets $g_{j} \in G$ and of the terms $t_{k} \in T$, respectively. Setting $y_{j k}=1$ means stating that a fragment $\mathcal{F}_{j k}$ with grouping set $G b y\left(\mathcal{F}_{j k}\right)=g_{j}$ and measures $\operatorname{Meas}\left(\mathcal{F}_{j k}\right)=t_{k}$ will be created. Setting $x_{i j k}=1$ means stating that, when answering $q_{i}$, the measures in Meas $\left(q_{i}\right) \cap t_{k}$ will be read from $\mathcal{F}_{j k}$. Thus, a solution at the same time denotes a fragmentation,

$$
\mathcal{H}=\left\{\mathcal{F}_{j k}: y_{j k}=1\right\}
$$

and specifies on which fragment(s) each query in $Q$ is assumed to be executed. Figure 5 shows the solution which represents fragmentation $\mathcal{H}_{5}$ proposed in the example in Section 3.1, featuring 3 fragments. The solution also denotes that, for instance, $q_{1}$ is executed on $\mathcal{F}_{1}$.

Definition 5 (VFP) Given a multidimensional scheme $\mathcal{D}$, a workload $Q$ on $\mathcal{D}$, a set of candidate grouping sets $C$ and $(\mathcal{C})$ for each cube scheme $\mathcal{C} \in \mathcal{D}$, 
Table 3

Notation employed for index sets in the IP formulation of VFP.

\begin{tabular}{c|l}
\hline $\mathcal{Q}$ & Index set of the queries in $Q$ \\
$\mathcal{G}$ & Index set of the grouping sets in $G$ \\
$\mathcal{T}$ & Index set of the terms in $T$ \\
$\mathcal{M}$ & Index set of the measures in $\bigcup_{\mathcal{C} \in \mathcal{D}}$ Meas $(\mathcal{C})$ \\
$\mathcal{G}_{i} \subseteq \mathcal{G}$ & Index set of the useful grouping sets for query $i \in \mathcal{Q}$ \\
$\mathcal{M}_{i} \subseteq \mathcal{M}$ & Index set of the measures in Meas $\left(q_{i}\right)$ \\
$\mathcal{T}_{j} \subseteq \mathcal{T}$ & Index set of the terms at grouping set $j \in \mathcal{G}$ \\
$\mathcal{Q}_{i j} \subseteq \mathcal{T}$ & Index set of the useful terms for query $i \in \mathcal{Q}$ at grouping set $j \in \mathcal{G}_{i}$ \\
$\mathcal{M}_{j s} \subseteq \mathcal{T}$ & Index set of the useful terms for measure $s \in \mathcal{M}$ at grouping set $j \in \mathcal{G}$ \\
\hline
\end{tabular}

and a constraint $B$ on disk space, VFP consists in determining, among the fragmentations of $\mathcal{D}$ which require no more than $B$ disk space, the one which minimizes the cost of workload $Q$.

Using the notation for index sets summarized in Table 3, the IP formulation of VFP is as follows:

$$
\begin{aligned}
& \operatorname{Cost}(V F P)=\operatorname{Min} \sum_{i \in \mathcal{Q}} \sum_{j \in \mathcal{G}_{i}} \sum_{k \in \in_{\mathcal{Q}} T_{i j}} c_{i j k} x_{i j k} \\
& \text { s.t. } \sum_{j \in \mathcal{G}_{i}} \sum_{k \in \mathcal{M} \tau_{j s}} x_{i j k} \geq 1 \quad i \in \mathcal{Q}, s \in \mathcal{M}_{i} \\
& \sum_{k \in \in_{M} \mathcal{T}_{j s}} y_{j k} \leq 1 \quad j \in \mathcal{G}, s \in \mathcal{M} \\
& x_{i j k} \leq y_{j k} \quad i \in \mathcal{Q}, j \in \mathcal{G}_{i}, k \in{ }_{Q} \mathcal{T}_{i j} \\
& \sum_{j \in \mathcal{G}} \sum_{k \in \mathcal{T}_{j}} b_{j k} y_{j k} \leq B \\
& x_{i j k} \in\{0,1\} \\
& y_{j k} \in\{0,1\} \\
& i \in \mathcal{Q}, j \in \mathcal{G}_{i}, k \in{ }_{Q} \mathcal{T}_{i j} \\
& j \in \mathcal{G}, k \in \mathcal{T}_{j}
\end{aligned}
$$

where:

- $\operatorname{Cost}(V F P)$ denotes the optimal cost of VFP, i.e. the cost of $Q$ on the optimal fragmentation;

- $x_{i j k}=1 \mathrm{iff}$, when answering query $i$, the measures in term $k$ are read at grouping set $j$

- $y_{j k}=1$ iff $x_{i j k}=1$ for at least one query $i$;

- $c_{i j k}$ is the cost for (partially) answering query $i$ on fragment $\mathcal{F}_{j k}$ defined by $g_{j}$ and $t_{k}$, i.e. for reading the relevant term $k$ on grouping set $j$ (estimated for instance as in formula (A.2) in Appendix A); 
- $b_{j k}$ is the disk space required to store fragment $\mathcal{F}_{j k}$ (calculated as in formula (A.1) in Appendix A).

- Given $i \in \mathcal{Q}, \mathcal{G}_{i}=\left\{j: G b y\left(q_{i}\right) \preceq g_{j} \wedge\left(\exists \mathcal{C} \in \mathcal{D}: g_{j} \in \operatorname{Cand}(\mathcal{C}) \wedge \operatorname{Meas}\left(q_{i}\right) \cap\right.\right.$ $\operatorname{Meas}(\mathcal{C}) \neq \emptyset)\}$ is the index set of the grouping sets which might be used to solve $q_{i}$.

- Given $j \in \mathcal{G}$ and $s \in \mathcal{M},{ }_{M} \mathcal{T}_{j s}=\left\{k: m_{s} \in t_{k} \wedge\left(\exists \mathcal{C} \in \mathcal{D}: g_{j} \in \operatorname{Cand}(\mathcal{C}) \wedge\right.\right.$ $\left.\left.m_{s} \in \operatorname{Meas}(\mathcal{C})\right)\right\}$ is the index set of the terms which might be used to retrieve $m_{s}$ at grouping set $g_{j}$.

- Given $i \in \mathcal{Q}$ and $j \in \mathcal{G}_{i}, \hat{Q}_{i j}=\bigcup_{s \in \mathcal{M}_{i}} \mathcal{T}_{j s}$ is the index set of the terms which might be used to solve $q_{i}$ at grouping set $g_{j}$.

- Given $j \in \mathcal{G}$, the index set of the terms at grouping set $g_{j}$ is $\mathcal{T}_{j}=\bigcup_{i \in \mathcal{Q} Q} \mathcal{T}_{i j}=$ $\bigcup_{s \in \mathcal{M}} \mathcal{T}_{j s}$.

The explanation of the elements of formulation VFP is as follows. The objective function (6) states that the solution must minimize the sum of the costs for answering all queries in $Q$ on each fragment required (see Appendix A). Inequalities (7) impose that each measure required by a query is read on at least one grouping set, i.e. that the solution satisfies the workload. Inequalities (8) require that, for each grouping set, a measure belongs to at most one term, i.e. that the fragmentation is non-redundant as stated in property (3) of Definition 4. Inequalities (9) link the $x$ and $y$ variables and inequality (10) is the memory knapsack constraint, stating that the disk space required by fragmentation must be less than B. Finally, (11) and (12) are the integrality constraints. As to the other fragmentation properties, consistency (1) is implicitly expressed by formulating the IP constraints on the restricted index sets $\mathcal{G}_{i}, \mathcal{Q}_{i j}$, and ${ }_{M} \mathcal{T}_{j s}$ instead of their supersets $\mathcal{G}$ and $\mathcal{T}$. Lossless fragmentation (2) is enforced by inserting in the workload, for each cube scheme $\mathcal{C}$, a dummy query $q_{\mathcal{C}}$ such that $\operatorname{Meas}\left(q_{\mathcal{C}}\right)=\operatorname{Meas}(\mathcal{C}), G b y\left(q_{\mathcal{C}}\right)=G b y(\mathcal{C})$, and Freq $\left(q_{\mathcal{C}}\right)=0$ : this trick allows for making fragmentation lossless without altering the workload cost.

Notice the structure of VFP. There are essentially three blocks of constraints: inequalities (7), (8) and (10). Inequalities (7), the query satisfaction constraints, are actually set covering constraints imposing that exactly one $x_{i j k}$ variable enters the solution for each $i$ and for each measure in $\mathcal{M}_{i}$ : problem costs are attached only to variables $x_{i j k}$, thus this set covering part directly affects the objective cost. Inequality (10), as mentioned, is a knapsack constraint, while inequalities (8) are needed to impose measure-term surjectivity in the form of set packing constraints. Problem VFP is NP-hard, since when no constraint is imposed on memory (i.e., $B$ is very big) and only one query $i \in \mathcal{Q}$ is defined for each $j \in \mathcal{G}_{i}$ and $k \in{ }_{Q} \mathcal{T}_{i j}$, it becomes a standard set covering problem, which is NP-hard. When memory is tight, VFP is further complicated by the interplay of set covering, set packing and knapsack.

By a linear relaxation of integrality constraints, that is by substituting con- 
Table 4

Workload composition in terms of number of queries on each cube scheme.

\begin{tabular}{c|ccc|c}
\hline Workload & on $L I$ & on $P S$ & on $L I$ and $P S$ & Total \\
\hline$Q_{1}$ & 15 & 2 & 3 & 20 \\
$Q_{2}$ & 19 & 6 & 5 & 30 \\
$Q_{3}$ & 23 & 9 & 8 & 40 \\
\hline
\end{tabular}

straints (11) and (12) with:

$$
\begin{array}{ll}
x_{i j k} \leq 1 & i \in \mathcal{Q}, j \in \mathcal{G}_{i}, k \in \hat{Q}_{i j} \\
y_{j k} \leq 1 & j \in \mathcal{G}, k \in \mathcal{T}_{j}
\end{array}
$$

we get problem LVFP: a linear problem that can be solved in polynomial time and whose optimal solution value $\operatorname{Cost}(L V F P)$ constitutes a lower bound to $\operatorname{Cost}(V F P)$, the optimal solution cost of problem VFP.

\section{Experimental results}

An extensive set of tests have been carried out in order to verify the effectiveness of fragmentation. Tests are aimed at comparing our approach to the all-or-nothing solution to view materialization and at emphasizing the specific characteristics of the fragmented solutions. Tests are based on the well-known TPC-H benchmark [20], sized at 1 GB and loaded on Red Brick Decision Server 6.0. The operational scheme proposed within TPC-H has been used to obtain the two cube schemes, $L I$ and $P S$, whose simplified versions have been used as working example in the previous sections. In our implementation, $L I$ consists of 7 hierarchies with 40 attributes and 10 measures, PS of 3 hierarchies with 20 attributes and 3 measures.

The tests are organized according to three different workloads with increasing size. Table 4 reports the distribution of queries on the different cube schemes: the bulk of the queries works on $L I$, while the percentage of drill-across queries slightly increases from $15 \%$ to $20 \%$. $Q_{1}$ tightly models the TPC-H benchmark including all the queries that can be formulated as GPSJ expressions, as well as the GPSJ subqueries that are contained in non-GPSJ ones. $Q_{2}$ and $Q_{3}$ extend TPC-H by adding new queries with different grouping sets and whose measures are chosen consistently with the application logic defined in the benchmark.

Maintaining a realistic clustering of measures in the test workloads is impor- 
Table 5

Average and standard deviation (in parentheses) of the affinity support for the three workloads.

\begin{tabular}{c|ccc|c}
\hline Workload & on $L I$ & on $P S$ & on $L I$ and $P S$ & Average \\
\hline$Q_{1}$ & $26 \%(33 \%)$ & $17 \%(33 \%)$ & $13 \%(32 \%)$ & $23.1 \%(32.8 \%)$ \\
$Q_{2}$ & $20 \%(31 \%)$ & $27 \%(31 \%)$ & $26 \%(41 \%)$ & $22.4 \%(32.7 \%)$ \\
$Q_{3}$ & $21 \%(37 \%)$ & $26 \%(22 \%)$ & $22 \%(28 \%)$ & $22.3 \%(31.8 \%)$ \\
\hline
\end{tabular}

Table 6

Average and standard deviation (in parentheses) of the affinity confidence for the three workloads.

\begin{tabular}{c|ccc|c}
\hline Workload & on $L I$ & on $P S$ & on $L I$ and $P S$ & Average \\
\hline$Q_{1}$ & $4 \%(4 \%)$ & $7 \%(14 \%)$ & $8 \%(19 \%)$ & $4.9 \%(7.2 \%)$ \\
$Q_{2}$ & $3 \%(12 \%)$ & $11 \%(12 \%)$ & $12 \%(19 \%)$ & $6.1 \%(13.2 \%)$ \\
$Q_{3}$ & $3 \%(13 \%)$ & $9 \%(7 \%)$ & $8 \%(4 \%)$ & $5.3 \%(9.8 \%)$ \\
\hline
\end{tabular}

tant to avoid to excessively emphasize the effect of fragmentation. On the other hand, measures are naturally clustered by the level of correlation of the information they carry. The level of affinity of a couple of measures, $m_{i}$ and $m_{j}$, within workload $Q$ can be evaluated computing the support and the confidence for the association rule that relates the presence of one measure within a query with the presence of another measure (i.e. $m_{1} \Rightarrow m_{2}$ ):

$$
\begin{aligned}
& \operatorname{supp}\left(m_{i}, m_{j}\right)=\frac{\left|\left\{q \in Q: m_{i} \in \operatorname{Meas}(q) \wedge m_{j} \in \operatorname{Meas}(q)\right\}\right|}{|Q|} \\
& \operatorname{conf}\left(m_{i}, m_{j}\right)=\frac{\left|\left\{q \in Q: m_{i} \in \operatorname{Meas}(q) \wedge m_{j} \in \operatorname{Meas}(q)\right\}\right|}{\left|\left\{q \in Q: m_{i} \in \operatorname{Meas}(q)\right\}\right|}
\end{aligned}
$$

An estimate of the global affinity level for workload $Q$ on cube scheme $\mathcal{C}$ is given by the average support and confidence for all the couples of measures in Meas $(\mathcal{C})$. A high value of the support denotes that measures appear clustered together in most queries; given the support, the confidence is high if measures appear in different clusters only within a few queries. Tables 5 and 6 show the average and standard deviation of, respectively, support and confidence of the global affinity for the three workloads; data are shown separately for the different cube schemes.

In the following subsections, the workload execution cost is expressed in terms of disk pages. It is remarkable that all the data presented in the tests were directly measured on the DBMS rather than obtained from simulations, thus avoiding that the simplifications induced by the cost function proposed in Appendix A invalidate our results. As to indexing of data, we adopted a standard solution which provides a $\mathrm{B}^{+}$-Tree index on each primary key of both fact and dimension tables. 
Table 7

Shift from optimality induced by the heuristic reduction of the set of candidate views on the materialization solution for $Q_{1}$ with $1.4 \mathrm{~GB}$ available.

\begin{tabular}{c|rrcr}
\hline \multirow{2}{*}{ Numb. of Views } & \multicolumn{2}{|c}{ Cost } & \multicolumn{2}{c}{ Shift from optimality } \\
\cline { 2 - 5 } & Unfrag. & Frag. & Unfrag. & Frag. \\
\hline 100 & 3286453 & 538372 & $256 \%$ & $6 \%$ \\
300 & 1284958 & 520647 & $0 \%$ & $2 \%$ \\
500 & 1284958 & 510230 & $0 \%$ & $0 \%$ \\
743 & 1284958 & 510230 & $0 \%$ & $0 \%$ \\
\hline
\end{tabular}

The algorithms for view materialization and fragmentation are implemented within WAND [7], the data warehouse design tool we developed. In selecting the all-or-nothing approach to materialization to be compared with fragmentation, we observed that: (1) all approaches to materialization are characterized by an objective function to be optimized and an optimization algorithm; (2) any objective function could be adopted in principle, but the comparison is sound only if the same function used for fragmentation is adopted; (3) as to the optimization algorithm, all approaches to materialization we are aware of are based on heuristic algorithms which deliver sub-optimal solutions [25]. Thus, adopting one or the other of the materialization algorithms proposed in the literature would not alter significantly the comparison results: the one we implemented is described in [1] and selects views using a tabu search heuristics; the objective function is the one described in Appendix A, which estimates the execution cost of the workload.

Solving the fragmentation problem on the full $C$ and $(\mathcal{C})$ set becomes unfeasible for large workloads; the solution we adopted, proposed in [1], preliminarily reduces the cardinality of each $C$ and $(\mathcal{C})$ by repeatedly dropping the candidate views whose grouping set is very close to the one of another candidate view in $\operatorname{Cand}(\mathcal{C})$. The reason for this heuristic criterion, that is well-known and accepted by data warehouse designers [14], relies on the observation that it is useless to materialize fragments/views on very similar grouping sets. When comparing fragmented solutions to unfragmented ones, the same starting set of candidate views is always used. Table 7 shows how the preliminar reduction of the candidate view sets affects the solution optimality. All test has been carried out on $Q_{1}$ and show that this heuristic criterion actually degrades the solution only in the all-or-nothing approach when dramatic reductions are operated. It is remarkable that, even when significant reductions are applied, the extra flexibility induced by fragmentation limits the shift from optimality. 


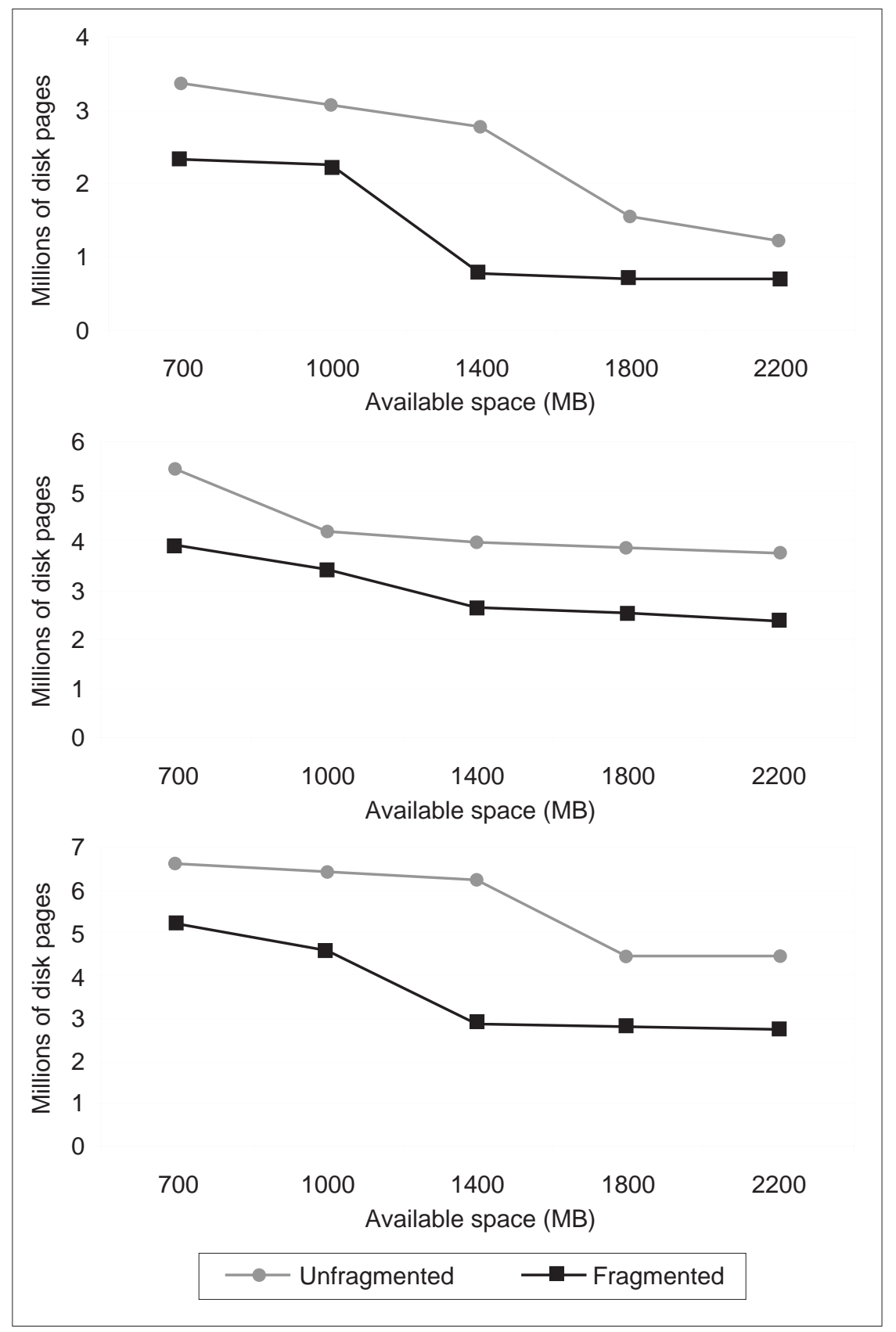

Fig. 6. Fragmented and unfragmented workload costs for $Q_{1}$ (top), $Q_{2}$ (middle), and $Q_{3}$ (bottom) varying the space constraints.

\subsection{Workload cost optimization}

The aim of this set of tests is to analyze to which extent fragmentation reduces the workload cost and which factors determine the reduction. Figure 6 compares the execution costs for the fragmented and unfragmented solution of $Q_{1}, Q_{2}$, and $Q_{3}$. For each workload, the space constraint ranges from 700 $\mathrm{MB}$ to $2200 \mathrm{MB}$; since the primary views globally require $640 \mathrm{MB}$, the space 


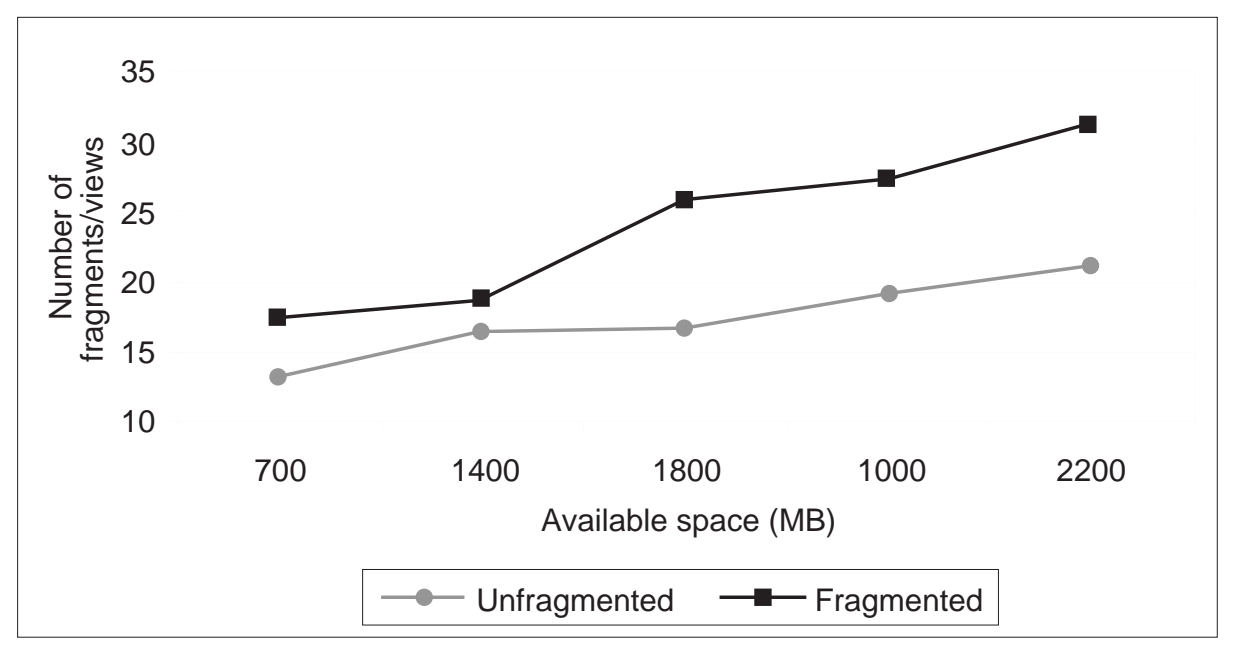

Fig. 7. Number of fragments and views materialized for different disk space constraints (data are averaged on the three workloads).

reserved for secondary views varies from $60 \mathrm{MB}$ to $1560 \mathrm{MB}$.

The cost reduction, that ranges from $18 \%$ to $73 \%$ and is $38 \%$ in the average, is due to two interrelated factors: on the one side, each query requires less disk pages to be answered since only relevant measures are stored in fragments; on the other, the saved space can be used to materialize more fragments which further enhance performances. It should be noted that the previously mentioned factors participate differently in reducing the cost, according to the available space. When only a limited space is available for materialization, most of the saving comes from the reduced size of fragments while, when more space is available, the saving is mainly due to the execution of queries on fragments that do not fit the disk space constraint in the all-or-nothing solution. The rationale for this behavior is that, the more the materialized views, the more the disk space saved by dropping unuseful measures, the more the extra fragments materialized; this is confirmed by Figure 7 which compares the number of fragments and the number of views for different space constraints, showing that the former increases more quickly than the latter. Table 8 shows, for workload $Q_{1}$ and for different space constraints, the number of queries which in both the fragmented and the unfragmented solutions are solved on the same grouping set and the percentage of saving due to measure dropping.

Fragmentation also allows to store within the same fragment measures from different cube schemes. Table 9 shows the number of queries executed on such unified fragments for different workloads and space constraints. It should be noted that, for some drill-across queries, no unified fragment may be available due to the lack of disk space which forces the DBMS to execute the query on one or more different (sub-optimal) fragments. In other cases, unified fragments are not materialized since it is not convenient at all, for example when other queries can be solved using a subset of the measures to be unified. In 
Table 8

Number of queries of $Q_{1}$ solved on the same grouping set in both the fragmented and unfragmented solution, and percentage saving due to measure dropping.

\begin{tabular}{c|cc}
\hline $\begin{array}{c}\text { Available } \\
\text { space }\end{array}$ & $\begin{array}{c}\text { Number } \\
\text { of queries }\end{array}$ & $\begin{array}{c}\text { Saving due to } \\
\text { measure dropping }\end{array}$ \\
\hline 700 & 13 & $95 \%$ \\
1000 & 13 & $30 \%$ \\
1400 & 10 & $5 \%$ \\
1800 & 11 & $12 \%$ \\
2200 & 14 & $2 \%$ \\
\hline
\end{tabular}

Table 9

Number of queries executed on unified fragments for the three workloads.

\begin{tabular}{c|ccc}
\hline Available space & $Q_{1}$ & $Q_{2}$ & $Q_{3}$ \\
\hline 700 & 0 & 4 & 4 \\
1000 & 0 & 4 & 4 \\
1400 & 0 & 5 & 3 \\
1800 & 1 & 4 & 3 \\
2200 & 1 & 4 & 3 \\
\hline
\end{tabular}

all these cases, unification is driven by the cost function, thus the solution obtained is always optimized for the given workload and disk space constraint.

In principle, within the optimal materialization solution, when no constraints on space are posed, each query in the workload should be answered on a view with the same grouping set required by the query. In the all-or-nothing approach, this is achieved by materializing one view for each grouping set required by at least one query; in the fragmented approach, materializing only the subset of relevant measures will suffice. Consequently, as confirmed at first glance by analyzing the graphs, fragmented curves flatten for smaller disk space than unfragmented ones. When the space constraint is not fixed a priori and is left to the designer's choice, the best trade-off between performance and space should be determined by a threshold on the extra-space benefit (i.e. such that the cost decreases for each MB further devoted to materialization); in the fragmented approach, such threshold will be crossed for lower disk spaces.

Although it is very likely that the core workload is available to the designer a priori, it is not possible to know in advance all the queries that will be submitted to the warehouse. Extemporary queries formulated by the users during OLAP sessions may decrease the global saving induced by the vertical fragmentation solution which is tailored on a specific workload. The effects 
Table 10

Cost of a workload when executed on the materialization solution optimized for itself and for $Q_{1}$ (with space constraint $1 \mathrm{~GB}$ ).

\begin{tabular}{c|cccc}
\hline \multirow{2}{*}{ Mat. solution } & \multicolumn{2}{|c}{$Q_{2}$} & \multicolumn{2}{c}{$Q_{3}$} \\
\cline { 2 - 5 } & Opt. for $Q_{2}$ & Opt. for $Q_{1}$ & Opt. for $Q_{3}$ & Opt. for $Q_{1}$ \\
\hline Unfragmented & 4138 & 12989 & 6424 & 49537 \\
Fragmented & 3411 & 11204 & 4565 & 52772 \\
\hline
\end{tabular}

of extemporary queries have been experimentally evaluated by comparing the performances of the unfragmented and fragmented solutions on workloads containing also queries not included in the reference workloads used for optimization. In particular, in Table 10 we compare the costs for executing $Q_{2}\left(Q_{3}\right)$ on the (fragmented and unfragmented) materialization solutions optimzed for $Q_{2}\left(Q_{3}\right)$ and for $Q_{1}$, respectively. It is apparent that, as we might expect, the workload cost rises quickly for both the fragmented and unfragmented solutions as soon as extemporary queries are considered. When increasing their percentage, the performance for the fragmented solution decays a little more quickly than that of the unfragmented solution: in our tests, the two costs are comparable when $40 \%$ of the queries are extemporary. The reason for this behavior is that, though the extra-measures included in each view can be potentially useful to solve extemporary queries, its grouping set is often too coarse. Furthermore, the probability that a view can be used to answer a query increases for views with finer grouping sets which, unfortunately, determine lower cost savings.

\subsection{Considerations on storage space}

One of the main objections often moved to vertical fragmentation is that it causes a waste of disk space due to replication of keys. We wish to emphasize that our approach is based on reducing the size of fact tables by decreasing the number of measures included, while key replication occurs as a side effect only when multiple fragments are materialized on the same cube scheme and on the same grouping set. This is confirmed by the experimental results reported in this section, where the incidence of key replication on fragmentation effectiveness is evaluated.

Figure 8 reports the average number of surrogate keys composing the primary key of fragments. Most dimensions are completely grouped, which proves that fragments are, in the average, much coarser than primary views. In fact:

- OLAP queries are intrinsically aggregated in order to allow summary information to be easily analyzed by a human user. 


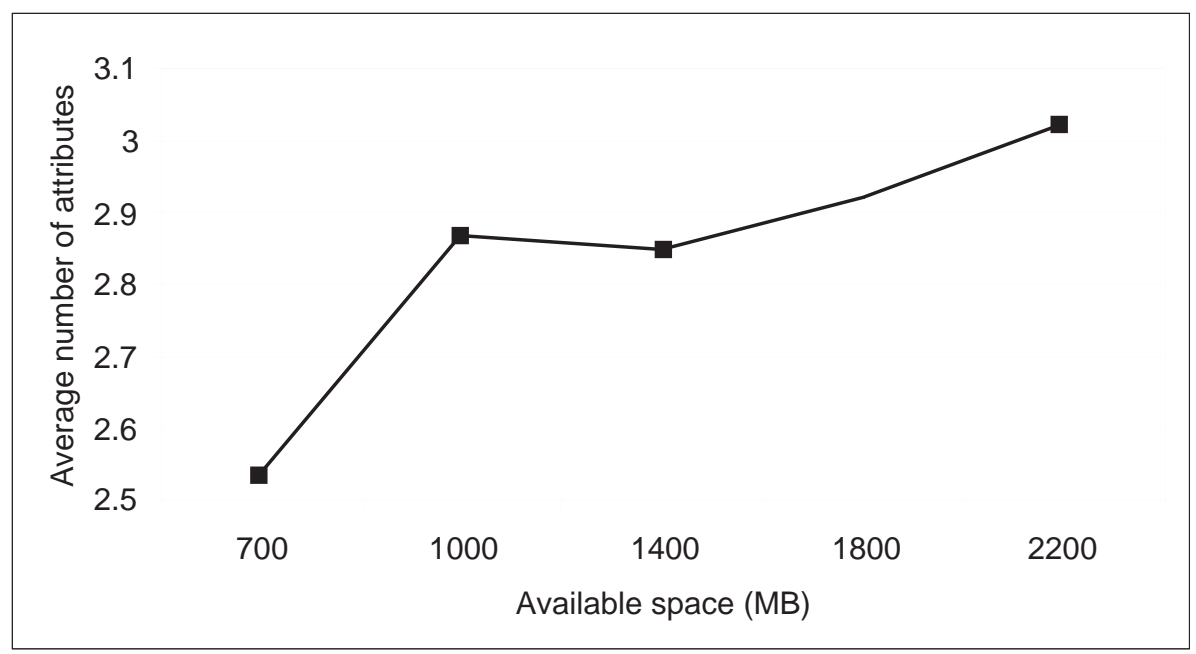

Fig. 8. Average number of surrogate keys for fragment (data are averaged on the three workloads).

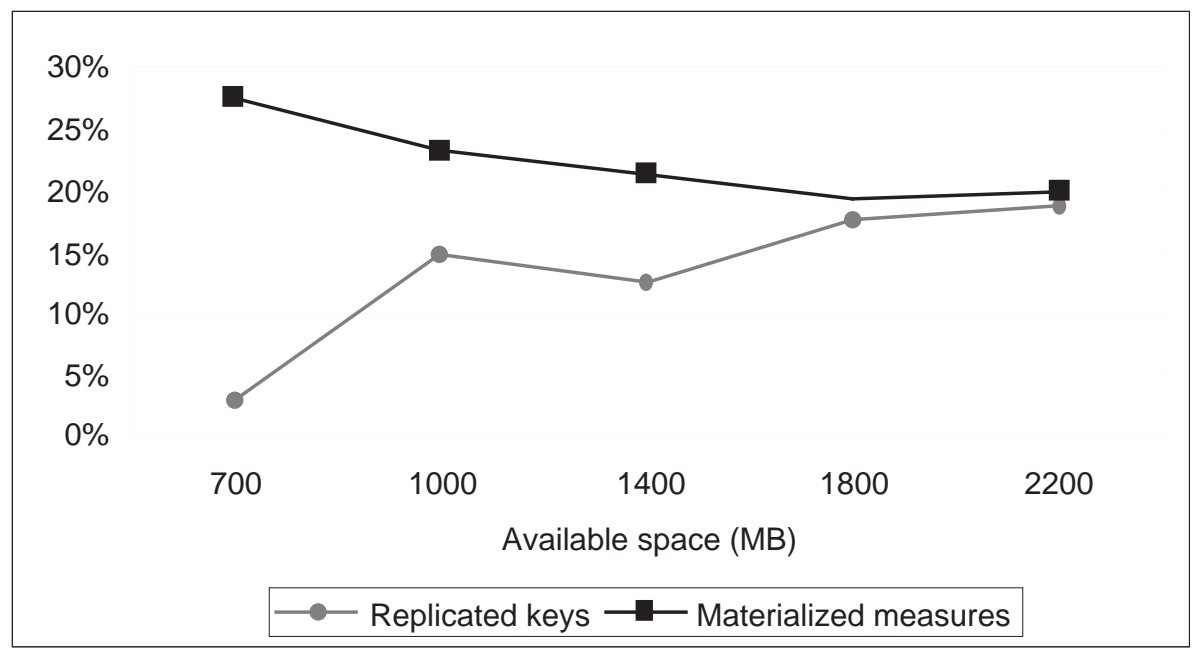

Fig. 9. Percentage of replicated keys and percentage of measures (data are averaged on the three workloads).

- The cost reduction induced by materialization is due to the reduced number of tuples that are read when answering a query on a view. On the other hand, due to the sparsity of primary views, only coarse grouping sets actually produce a significant reduction of the cardinalities of secondary views. Thus, most views are materialized on very coarse grouping sets.

The shortness of surrogate keys reduces the effects of redundancy since the space devoted to them is percentually lower for coarse fragments. As already stated, the effects of redundancy are further smoothed since our approach is inclined to materialize one fragment on each grouping set rather than more fragments on the same one. This behavior is confirmed by Figures 9 and 7 showing that the increase in the number of fragments is much faster than the increase in the number of replicated keys when rising the space constraint. The 
percentage of replicated keys (calculated as the fraction of the total number of attributes in the fact tables) is lower for tight constraints and never exceeds $19 \%$.

Figure 9 also shows the average percentage ratio between the number of measures materialized in the fragmented and in the unfragmented solutions, which decreases when more space is available since fragments become more specialized (i.e. few queries are answered on each fragment).

\subsection{Computational testing}

We implemented an exact methodology for solving instances of VFP. The computational testing was performed using a commercial IP solver (CPLEX 6.6 by Ilog Inc.) to produce first an optimal LVFP solution, thus a lower bound, and then the optimal integer solution. All tests were run on an Intel platform with $512 \mathrm{Mb}$ of RAM, running at $933 \mathrm{MHz}$ under Windows Me. We first generated some simple instances to fine-tune the CPLEX parameters and then solved the instances described above. A full account of the computational testing can be found in Table 11. The columns show the following data:

- Probl: problem identifier;

- $n$ : number of queries in the workload;

- $B$ : size of memory constraint;

- $\operatorname{Cost}(L V F P)$ : optimal linear relaxation solution cost;

- err: percentage distance from IP optimality of Cost $(L V F P)$;

- $t(L V F P):$ CPU time to obtain Cost $(L V F P)$;

- $\operatorname{Cost}(V F P)$ : optimal integer solution cost;

- $t(V F P):$ CPU time to obtain $\operatorname{Cost}(V F P)$;

Problem instances Qa to Qe are used to tune the final algorithm and can all be easily solved by CPLEX. The other instances are those described in the previous sections. Besides the increase in their dimension, it is apparent from column $t(V F P)$ how they are structurally more complex than the training ones. As expected, the tighter the memory constraints, the harder the instance. This can be seen both from the higher error of the bound w.r.t. the optimal IP cost and from the time needed to get the optimal IP solution. Instances Q2S7 and Q3S7 are the hardest ones, they could be solved with the available RAM, but the CPU time needed is huge. This increased difficulty arises from the interplay of all problem constraints, whereas with loose memory requirements the active constraints are essentially the set covering ones.

All problem instances used in this presentation can be downloaded from the URL: http://astarte.csr.unibo.it/data/vfp. 
Table 11

Computational test summary results.

\begin{tabular}{|c|c|c|c|c|c|c|c|}
\hline Probl & $n$ & $B$ & $\operatorname{Cost}(L V F P)$ & $e r r$ & $t(L V F P)$ & $\operatorname{Cost}(V F P)$ & $t(V F P)$ \\
\hline $\mathrm{Qa}$ & 3 & 1400 & 50475 & 0.00 & 0.05 & 50475 & 0.00 \\
\hline $\mathrm{Qb}$ & 5 & 1400 & 281816 & 2.13 & 0.22 & 287957 & 0.00 \\
\hline Qc & 10 & 1400 & 65190 & 0.00 & 0.66 & 65190 & 0.00 \\
\hline $\mathrm{Qd}$ & 10 & 1400 & 33976 & 0.00 & 0.06 & 33976 & 0.00 \\
\hline $\mathrm{Qe}$ & 10 & 1400 & 116463 & 0.00 & 0.11 & 116463 & 0.00 \\
\hline Q1S7 & 20 & 700 & 322535 & 13.77 & 63.65 & 374047 & 847.94 \\
\hline Q1S10 & 20 & 1000 & 152816 & 0.70 & 904.95 & 153894 & 5308.99 \\
\hline Q1S14 & 20 & 1400 & 98046 & 1.16 & 306.48 & 99201 & 3327.66 \\
\hline Q1S18 & 20 & 1800 & 96270 & 0.00 & 342.41 & 96270 & 52.73 \\
\hline Q1S22 & 20 & 2200 & 96270 & 0.00 & 370.59 & 96270 & 31.30 \\
\hline $\mathrm{Q} 2 \mathrm{~S} 7$ & 30 & 700 & 564786 & 11.60 & 346.97 & 638882 & 24563.01 \\
\hline Q2S10 & 30 & 1000 & 353786 & 0.47 & 1343.03 & 355456 & 4738.76 \\
\hline Q2S14 & 30 & 1400 & 274042 & 2.14 & 645.87 & 280029 & 6880.08 \\
\hline Q2S18 & 30 & 1800 & 248205 & 1.94 & 513.66 & 253117 & 1677.59 \\
\hline $\mathrm{Q} 2 \mathrm{~S} 22$ & 30 & 2200 & 241330 & 0.00 & 607.91 & 241330 & 162.80 \\
\hline Q3S7 & 40 & 700 & 718601 & 10.14 & 1824.24 & 799666 & 76703.28 \\
\hline Q3S10 & 40 & 1000 & 464241 & 0.06 & 653.83 & 464535 & 8539.98 \\
\hline Q3S14 & 40 & 1400 & 376146 & 1.78 & 551.29 & 382947 & 3780.41 \\
\hline Q3S18 & 40 & 1800 & 336431 & 1.37 & 680.53 & 341090 & 4362.62 \\
\hline Q3S22 & 40 & 2200 & 316795 & 0.11 & 668.17 & 317155 & 2561.45 \\
\hline
\end{tabular}

\section{Related work}

The problem of determining the optimal partitioning given a workload has been widely investigated within the context of centralized as well as distributed database systems, considering non-redundant allocation of fragments (for instance, see $[3,15,19])$; unfortunately, the results reported in the literature cannot be applied here since the redundancy introduced by materializing views at different aggregation levels binds the partitioning problem to that of deciding on which view(s) each query should be executed. Thus, ad-hoc approaches must be devised for MDs.

While horizontal partitioning has been widely investigated and is currently 
adopted by designers [8], to the best of our knowledge only a few approaches to vertical fragmentation in MDs have been devised. In [17], fragmentation is extended to both measures and non-key dimensions (i.e. dimensions functionally determined by other dimensions); on the other hand, no algorithm for determining the optimal fragmentation is proposed. In [4], views are partitioned vertically in order to build dataindexes to enhance performance in parallel implementations of MDs; still, no suggestion is given on how to determine the optimal partitioning. In [5], materialization and fragmentation are seen as separate steps; the views are determined by the materialization algorithm and then fragmented, thus compromising the process overall optimality.

At the physical level, vertical fragmentation is partially implemented by projection indexes that store a single column of a relational table [18]; the correspondence between the rows of the index and the rows in the main table is transparently maintained by the DBMS. The rationale for this kind of index, which is particularly useful for selection and aggregation queries, is that scanning a smaller structure is faster than scanning the whole table. Though projection indexes are usually considered as auxiliary structures to improve retrieval efficiency, the SYBASE IQ commercial DBMS [26,23] pushes their use to the limit by replacing the data themselves, i.e. by storing data by columns instead of rows. Thus, in practice, SYBASE implements an extreme fragmentation in which each fragment includes a single measure. In this case, since the correspondence between values belonging to the same row is maintained by row identifiers, fragmentation does not require the replication of the key attributes.

Currently, to the best of our knowledge, no DBMS allows to include multiple columns into one projection index, thus our fragmentation technique cannot be applied at the physical level. However, given the competitive performance of SYBASE, it is easy to predict a relevant utility for a workload-based fragmentation if multi-attribute projection indexes will be implemented. In this case, the optmimal fragmentation could be determined using our approach by considering, in the function estimating the access costs, that keys are not replicated.

\section{Conclusions}

In this paper we have proposed a technique for materializing views in vertical fragments, aimed to tightly fit the reference workload. In the context of multidimensional databases, due to both the redundancy of data guaranteed by materialization and to the nature of queries, fragmentation is more complex than for traditional relational databases on the one hand, on the other it may yield more significant benefits. 
The experimental tests suggest the effectiveness of our approach in reducing the workload cost as compared to the classical approach to view materialization. The robustness of fragmentation has been evaluated even with reference to extemporary queries. The tests also allowed to confute the main drawback typically ascribed to fragmentation, i.e. the replication of keys, showing that its impact on the storage requirements is not relevant. Besides, key replication could be completely avoided when applying fragmentation at the physical level, implementing it for instance by means of multi-attribute projection indexes.

\section{A The cost function}

Among all the feasible solutions to the fragmentation problem, we are interested in the one which minimizes the cost for executing the workload. We believe it is convenient to keep logical design independent on access plans in order to both provide a more general solution and reduce complexity. Thus, the cost function we propose intentionally abstracts from any assumptions on the access paths, being based on the number of disk pages in which the tuples of interest for a given query are stored.

Let $q_{i}$ be a query requiring at least one measure in fragment $\mathcal{F}_{j k}$ defined by $\operatorname{Gby}\left(\mathcal{F}_{j k}\right)=g_{j}$ and $\operatorname{Meas}\left(\mathcal{F}_{j k}\right)=t_{k}$, and $\operatorname{Freq}\left(q_{i}\right)$ be its frequency. The number of tuples of $\mathcal{F}_{j k}$ which must be accessed in order to answer $q_{i}$ is $\operatorname{Sel}\left(q_{i}\right) \cdot \operatorname{Card}\left(\mathcal{F}_{j k}\right)$, where $\operatorname{Card}\left(\mathcal{F}_{j k}\right)$ is the cardinality of $\mathcal{F}_{j k}$ estimated for instance as in [22]. The total number of pages in which $\mathcal{F}_{j k}$ is contained is

$$
b_{j k}=\left\lceil\frac{\operatorname{Card}\left(\mathcal{F}_{j k}\right)}{\beta_{j k}}\right\rceil
$$

where $\beta_{j k}$ is the number of tuples per disk page for $\mathcal{F}_{j k}$. The expected, frequency-weighted number of pages in which the tuples of $\mathcal{F}_{j k}$ necessary for $q_{i}$ are stored, $c_{i j k}$, can be estimated with the Cardenas formula $\Phi[2]$ as follows:

$$
c_{i j k}=\operatorname{Freq}\left(q_{i}\right) \cdot \Phi\left(\operatorname{Sel}\left(q_{i}\right) \cdot \operatorname{Card}\left(\mathcal{F}_{j k}\right), b_{j k}\right)
$$

With reference to the index set notation summarized in Table 3 , the cost of executing query $i$ against the fragmentation denoted by $X=\left\{x_{i j k}\right\}$ is then estimated as the total number of disk pages which must be accessed in order to retrieve all the measures in $q_{i}$ :

$$
\operatorname{Cost}_{i}(X)=\sum_{j \in \mathcal{G}_{i}} \sum_{k \in \in_{Q} T_{i j}} c_{i j k} x_{i j k}
$$


where $x_{i j k}=1$ if, when answering $q_{i}$, the measures in $\operatorname{Meas}\left(q_{i}\right) \cap t_{k}$ are read from $\mathcal{F}_{j k}, x_{i j k}=0$ otherwise. Finally, the overall cost for workload $Q$ is:

$$
\operatorname{Cost}(X)=\sum_{i \in \mathcal{Q}} \operatorname{Cost}_{i}(X)=\sum_{i \in \mathcal{Q}} \sum_{j \in \mathcal{G}_{i}} \sum_{k \in \in_{Q} T_{i j}} c_{i j k} x_{i j k}
$$

This is the cost function minimized in (6) within the IP formulation of VFP. Though the actual number of pages read when executing the workload may be higher depending on the access path followed, we believe that this function represents a good trade-off between generality and accuracy.

\section{References}

[1] E. Baralis, S. Paraboschi, and E. Teniente. Materialized view selection in multidimensional database. In Proc. 23rd $V L D B$, pages 156-165, Athens, Greece, 1997.

[2] A.F. Cardenas. Analysis and performance of inverted database structures. Communications of the ACM, 18(5):253-263, 1975.

[3] W.W. Chu and I.T. leong. A transaction-based approach to vertical partitioning for relational database system. IEEE Trans. on Software Engineering, $19(8): 804-812,1993$.

[4] A. Datta, B. Moon, and H. Thomas. A case for parallelism in data warehousing and OLAP. In Proc. IEEE First Int. Workshop on Data Warehouse Design and OLAP Technology, 1998.

[5] M. Golfarelli, D. Maio, and S. Rizzi. Applying vertical fragmentation techniques in logical design of multidimensional databases. In Proc. DaWaK 2000, Greenwich, UK, 2000.

[6] M. Golfarelli and S. Rizzi. View materialization for nested GPSJ queries. In Proc. DMDW'2000, Stockholm, 2000.

[7] M. Golfarelli and S. Rizzi. Wand: A case tool for data warehouse design. In Demo Proc. 17th ICDE, Heidelberg, Germany, 2001.

[8] V. Gopalkrishnan, Q. Li, and K. Karlapalem. Efficient query processing with associated horizontal class partitioning in an object relational data wa rehousing environment. In Proc. DMDW'2000, Stockholm, 2000.

[9] J. Gray, A. Bosworth, A. Lyman, and H. Pirahesh. Data-Cube: a relational aggregation operator generalizing group-by, cross-tab and sub-totals. Technical Report MSR-TR-95-22, Microsoft Research, 1995.

[10] M. Grötschel, R.L. Graham, and L. Lovász. Handbook of Combinatorics. Elsevier, 1995. 
[11] A. Gupta, V. Harinarayan, and D. Quass. Aggregate-query processing in datawarehousing environments. In Proc. 21rd VLDB, Zurich, Switzerland, 1995.

[12] M. Gyssens and L.V .S. Lakshmanan. A foundation for multi-dimensional databases. In Proc. 23rd VLDB, pages 106-115, Athens, Greece, 1997.

[13] V. Harinarayan, A. Rajaraman, and J. Ullman. Implementing data cubes efficiently. In Proc. ACM Sigmod Conf., Montreal, Canada, 1996.

[14] R. Kimball. The data warehouse toolkit. John Wiley \& Sons, 1996.

[15] X. Lin, M. Orlowska, and Y. Zhang. A graph-based cluster approach for vertical partitioning in database design. Data \&3 Knowledge Engineering, 11:151-169, 1993.

[16] V. Maniezzo and A. Colorni. The Ant System applied to the quadratic assignment problem. IEEE Trans. on Knowledge and Data Engineering, $11(5): 769-778,1999$.

[17] D. Munneke, K. Wahlstrom, and M. Mohania. Fragmentation of multidimensional databases. In Proc. 10th Australasian Database Conf., pages 153-164, Auckland, 1999.

[18] P. E. O'Neil and D. Quass. Improved query performance with variant indexes. In Proc. ACM Sigmod Conf., pages 38-49, Tucson, Arizona, 1997.

[19] M.T. Özsu and P. Valduriez. Principles of distributed database systems. Prentice-Hall Int. Editors, 1991.

[20] M. Poess and C. Floyd. New TPC Benchmarks for decision support and web commerce. ACM SIGMOD Record, 29(4), 2000.

[21] S.M. Sait and H. Youssef. Iterative computer algorithms with applications in engineering. IEEE Computer Society press, Piscataway, NJ, 1999.

[22] A. Shukla, P. Deshpande, J. Naughton, and K. Ramasamy. Storage estimation for multidimensional aggregates in the presence of hierarchies. In Proc. 22nd $V L D B$, pages 522-531, Mumbai, India, 1996.

[23] Sybase Inc. Adaptive Server IQ: Administration and performance Guide, 2001.

[24] D. Theodoratos and M. Bouzeghoub. A general framework for the view selection problem for data warehouse dsign and evolution. In Proc. DOLAP'O0, pages 1-8, Washington, DC, 2000.

[25] D. Theodoratos, S. Ligoudistianos, and T. Sellis. View selection for designing the global data warehouse. Data \& Knowledge Engineering, 39(3):219-240, 2001 .

[26] C. J. White. Sybase Adaptive Server IQ - A high-performance database for decision processing. Technical report, DataBase Associates International, Inc., 1999. 
[27] J. Yang, K. Karlaplem, and Q. Li. Algorithms for materialized view design in data warehousing environments. In Proc. 23rd VLDB, pages 136-145, Athens, Greece, 1997. 